\title{
An Analysis of the Vulnerability of Power Grids to Extreme Space Weather Using Complex Network Theory
}

\author{
R. Piccinelli and E. Krausmann \\ European Commission, Joint Research Center, Institute for the Protection and Security of the Citizen, Italy
}

\begin{abstract}
Space weather can affect critical infrastructures, causing damage to systems and resulting in failures or service disruptions. Of particular concern is the long-distance high-voltage power grid due to its vulnerability to geomagnetic storms. The induction of Geomagnetically Induced Currents (GICs) in the power network can damage equipment, push high-voltage transformers into their non-linear saturation range, or trip protection systems due to harmonics. These effects can lead to grid collapse.

Recently, several studies were commissioned in the U.S. to assess the power grid's vulnerability to extreme space weather and to investigate the potential consequences of prolonged blackouts on society. These studies highlighted a potentially major impact on the North American transmission grid and its components.

This study aims at identifying the vulnerability of the European power transmission grid with respect to extreme space weather by using complex network theory. We try to understand the spatial distribution and magnitude of GIC loading and the impact on grid operations potentially incurred. In a later step, this study will continue to estimate the impact of extreme space weather on society in Europe via the interdependencies of critical infrastructures with the power grid.
\end{abstract}

Keywords: Space Weather, Geomagnetic Induced Currents, Vulnerability, Complex Network Theory, Power Grids

\section{INTRODUCTION AND RATIONALE}

Space weather driven by solar disturbances can affect ground- and space-based critical infrastructures, potentially causing damage to systems and resulting in failures or service disruptions ${ }^{1}$. Of particular concern is the long-distance high-voltage power grid due to its vulnerability to geomagnetic storms that can develop when solar coronal mass ejections (CME) interact with the Earth's magnetosphere. The subsequent induction of Geomagnetically Induced Currents (GICs) in the power network can damage or destroy equipment, push high-voltage transformers into their non-linear saturation range, or trip protection systems due to harmonics. These effects lead to voltage instability that can eventually result in grid collapse ${ }^{2},{ }^{3}$.

The collapse of the Hydro-Quebec transmission network during the severe geomagnetic storm in 1989 was a wake-

1 Daglis, I.A. (ed.), Effects of space weather on technology infrastructure, Springer, ISBN 1-4020-2748-6 2005.

2 Krausmann E., Andersson E., Murtagh W. and Mitchison N., Space weather and power grids: findings and outlook, EUR 26370, ISBN 978-92-79-34812-9, 2013.

3 Schrijver, C.J. and Mitchell S.D., Disturbances in the US electric grid associated with geomagnetic activity, J. Space Weather and Space Climate, 3, A19, 2013. 
up call for the power industry world-wide and it is testimony to the vulnerability of the power grid to space weather effects. The Hydro-Quebec blackout also affected industrial production in a number of other sectors, drawing attention to the potential for significant ripple effects in case of a power outage ${ }^{4}$.

Recognizing the risks associated with the impact of a geomagnetic storm on the power grid, recently, several studies were commissioned in the U.S. to assess the power grid's vulnerability to extreme space weather and to investigate the potential consequences of prolonged blackouts on society $5,6,7,8$. These studies highlighted a potentially major impact on the North American transmission grid and its components, in particular high-voltage transformers. With manufacture lead times of 12 to 18 months, these grid elements would be the bottleneck for rapid recovery. Numerous other critical infrastructures would also be disrupted until grid operations could be re-established ${ }^{9}$.

An extreme geomagnetic storm such as the 1859 Carrington event, the largest geomagnetic storm on record, would encompass North America but also a significant part of Europe ${ }^{10}$. Various analyses on the European power grid were carried out regarding the reliability of the network in general ${ }^{11}$, or the vulnerability to local failures and intentional attacks $^{12},{ }^{13}$. However, hardly any information exists on the vulnerability of the European power grid to extreme space weather or the consequences of impact, including interdependencies with other critical infrastructures.

This study, which is work in progress, aims at identifying the vulnerability of the European power transmission grid with respect to extreme space weather by using complex network theory. We try to understand the spatial distribution and magnitude of GIC loading and the impact on grid operations potentially incurred. In a later step, this study will continue to estimate the impact of extreme space weather on society in Europe via the interdependencies of critical infrastructures with the power grid.

\section{METHODOLOGY}

When considering space weather effects on power networks, several issues have to be considered. For example, power grids are designed according to the $\mathrm{N}-1$ criterion which stipulates that the system should tolerate any single failure and still maintain its function ${ }^{14}$. Geomagnetic storms extend over vast geographic areas and their impact can involve wide portions of the electrical networks: it is therefore important to look beyond the $\mathrm{N}-1$ criterion and analyze the effects of several simultaneous failures over possibly spatially extended areas. Moreover, the geophysical characteristics of the location of the power grid also act as a source of vulnerability during space weather events: soil resistivity affects the propagation of magnetic disturbances resulting in induced large currents in long electrical transmission lines.

A vulnerability analysis therefore needs to adopt an approach which covers different perspectives, each dealing with the different aspects of power-grid vulnerability to extreme space weather. These perspectives are: global vulnerability

4 Boteler, D.H., Assessment of geomagnetic hazard to power systems in Canada, Natural Hazards, 23, 101-120, 2001.

5 National Research Council, Severe Space Weather Events-Understanding Societal and Economic Impacts, Workshop Report, ISBN 978-0-309-12769-1, 2008.

6 Kappenman J., Geomagnetic storms and their impacts on the US power grid, Meta - R- 319 Report, 2010.

7 JASON, Impact of severe space weather on the electric grid, JSR-11-320, The MITRE Corporation, 2011.

8 NERC, 2012 Special Reliability Assessment Interim Report: Effects of Geomagnetic Disturbances on the Bulk Power System, North American Electric Reliability Corporation, 2012.

9 National Research Council, Severe Space Weather Events-Understanding Societal and Economic Impacts, Workshop Report, ISBN 978-0-309-12769-1, 2008.

10 Pulkkinen A., Bernabeu E., Eichner J., Beggan C. and Thompson A.W.P., Generation of 100-year geomagnetically induced current scenarios, Space Weather, Vol. 10, S04003, 2012.

11 Brancucci Martinez-Anido C., Bolado R., De Vries L., Fulli G., Vandenbergh M. and Masera M., European power grid reliability indicators, what do they really tell?, Electric Power System Research, 90, 79-84, 2012.

12 Rosas-Casals M., Valverde S. and Solé R., Topological vulnerability of the European power grid under errors and attacks, Int. J. Bifurcation and Chaos, 17,2007.

13 Rosas-Casals M. and Solé R., Analysis of major failures in Europe's power grid, Electrical Power and Energy Systems, 33, 805808, 2011.

14 IRGC, White paper on managing and reducing social vulnerabilities from coupled critical infrastructures, International Risk Governance Council, Geneva, 2006. 
analysis, critical component analysis and geographical vulnerability analysis ${ }^{15}$. Global vulnerability analysis is carried out by exposing the system to strains of different intensity and orientation and estimating the negative consequences that arise. This is achieved, for example, by imposing different geomagnetic storm scenarios and estimating the resulting GICs in the system. Critical component analysis aims at estimating the consequences of single component failure or of a set of components failure, in order to identify those components that give rise to the largest negative consequences, i.e. those that are the most critical. Geographical vulnerability analysis consists of studying how space weather interacts with the geographic characteristics of the location of the system and of its components.

Vulnerability analysis requires the structure and behavior of the power grid to be modeled. There are many ways for doing this, depending on the physical and dynamical aspect of the system function to capture. Advanced models, such as those used in system engineering, are superior in capturing the physical behavior of the system but have a high computational cost for the kind of analysis carried out in these studies. In contrast, abstracted models, i.e. purely connectivity-based/topological models are used within the field of graph theory. They are computationally very fast but may not capture the relevant behavior of the system.

For modeling the electric power grid, a complex network theory approach has been chosen. The complex network approach distinguishes between a structural and a functional model of the system. Inspired by the field of network theory, the structural model captures the structural properties and consists of an abstract representation of the system: components of the power grid, for example bus-bars, are represented as nodes and connections; transmission lines, for example, are represented as edges. The functional model, which is inspired by system engineering, accounts for the physical properties and constraints of power systems and represents the response of the system when it is exposed to strains, such as currents caused by geomagnetic storms ${ }^{16}$.

This work proposes an approach that includes extended centrality measures to assess the structural vulnerability of power grids. It is intended as a first step to estimate the impact of extreme space weather effects on power grids and their potential consequences on interdependent critical infrastructures.

\section{PRELIMINARY RESULTS AND DISCUSSION}

GICs calculations are usually separated into two parts: a "geophysical part" which encompasses the determination of the electric field occurring at the Earth's surface due to CME interaction with the geomagnetic field, and an "engineering part" which covers the computation of GICs produced by the electric field ${ }^{17}$. In the following, only the calculation of the second part is illustrated; we assume that the electric field is already known.

Based on the physical model initially proposed by Lethinen and Pirjola ${ }^{18}$ and later by Viljanen et al. ${ }^{19}$, GICs flowing in the power grid to and from the Earth at the stations of a network having $N$ nodes are given by Eq. (1):

$$
\mathbf{I}_{\mathrm{e}}=\left(\mathbf{1}+\mathbf{Y}_{\mathrm{n}} \mathbf{Z}_{\mathrm{e}}\right)^{-1} \mathbf{J}_{\mathrm{e}}
$$

The NxN earthing impedance matrix $\mathbf{Z}_{\mathbf{e}}$ and the $\mathbf{N} \mathbf{N}$ network admittance matrix $\mathbf{Y}_{\mathbf{n}}$ includes the information on the system. The "perfect-earthing" current vector $\mathbf{J}_{\mathbf{e}}$ contains the information on the electric field, and $\mathbf{1}$ is the NxN unit identity matrix. When the earthing currents $\mathbf{I}_{\mathbf{e}}$ are known, the transmission line currents from node $i$ to node $\mathbf{j}, \mathbf{l}_{\mathrm{ij}}$, can be computed.

The Finnish 400-kV power grid configuration valid in October 1978 is used as benchmark test network, as proposed by Pirjola ${ }^{17}$. The test model power grid consists of 17 stations and 19 transmission lines. Each node includes a transformer ${ }^{17}$.

As a first scoping study, the behavior of each node with respect to GIC induction as function of the E-field orientation

15 Johansson J., Hassel H. and Cedergren A., Vulnerability analysis of interdependent critical infrastructures: case study of the Swedish railway system, International Journal of Critical Infrastructures, 7, No.4, 2011.

16 Johansson J., Hassel H. and Zio E., Reliability and vulnerability of critical infrastructures: comparing two approaches in the context of power systems, Reliability Engineering and System Safety, 120, 27-38, 2013.

17 Pirjola R., Properties of matrices included in the calculation of geomagnetically induced currents (GICs) in power systems and introduction of a test model for GIC computation algorithms, Earth Planet Space, 61, 263-272, 2009.

18 Lehtinen M. and Pirjola R., Currents produced in earthed conductor networks by geomagnetically-induced electric fields, Annales Geophysicae, 3, 4, 479-484, 1985.

19 Viljanen A., Pirjola R., Wik M., Adam A., Pracser E., Sakharov Y. and Katkalov J., Continental scale modelling of geomagnetically induced currents, J. Space Weather Space Climate, 2, A17, 2012. 
was analysed to study directional effects. All GIC calculations on the test system were carried out considering a uniform-magnitude field scenario which assumes an electric field strength of $1 \mathrm{~V} / \mathrm{km}$ which varies from north (zero degrees) to 165 degrees at 15 degree increments. Electric fields of identical magnitude separated by 180 degrees have identical effects, except for a GIC sign reversal, thus it is only necessary to simulate half of the spectrum of field orientations.

Figure 1 gives an overview of the result of the calculation of the GICs flowing into the system for each node: each color represents the value of the induced currents at different angles of orientation of the electric field, starting with the direction from north to south, at 0 degree (in blue), continuing in 15 degree increments to 165 degrees (in red). The green bar represents the east-west direction, corresponding to 90 degrees. The sign of the current refers to the direction of the flow: GICs exiting a node have positive values, GICs entering a node are negative. It is found that for nodes 7 to 13 the sign of the induced currents does not depend on the orientation of the field, while in other nodes, i.e., nodes 1 or 14, GICs change sign.

Since GICs flowing into the system represent a threat, it is of the utmost importance to understand which are the features of the network that influence the diffusion of GICs throughout the grid. One of the most important measures for understanding the role played by the components of the network is betweenness centrality ${ }^{20}$. The betweenness centrality of a node $i$ is defined as the fraction of shortest path between a pairs of nodes in the network that pass through $i$. When considering power networks, betweenness centrality can be regarded as a measure of the extent to which a vertex has control over the current flowing between other nodes. In power networks, however, GICs do not flow only along geodesic (shortest) paths because operational and physical rules drive the flow.

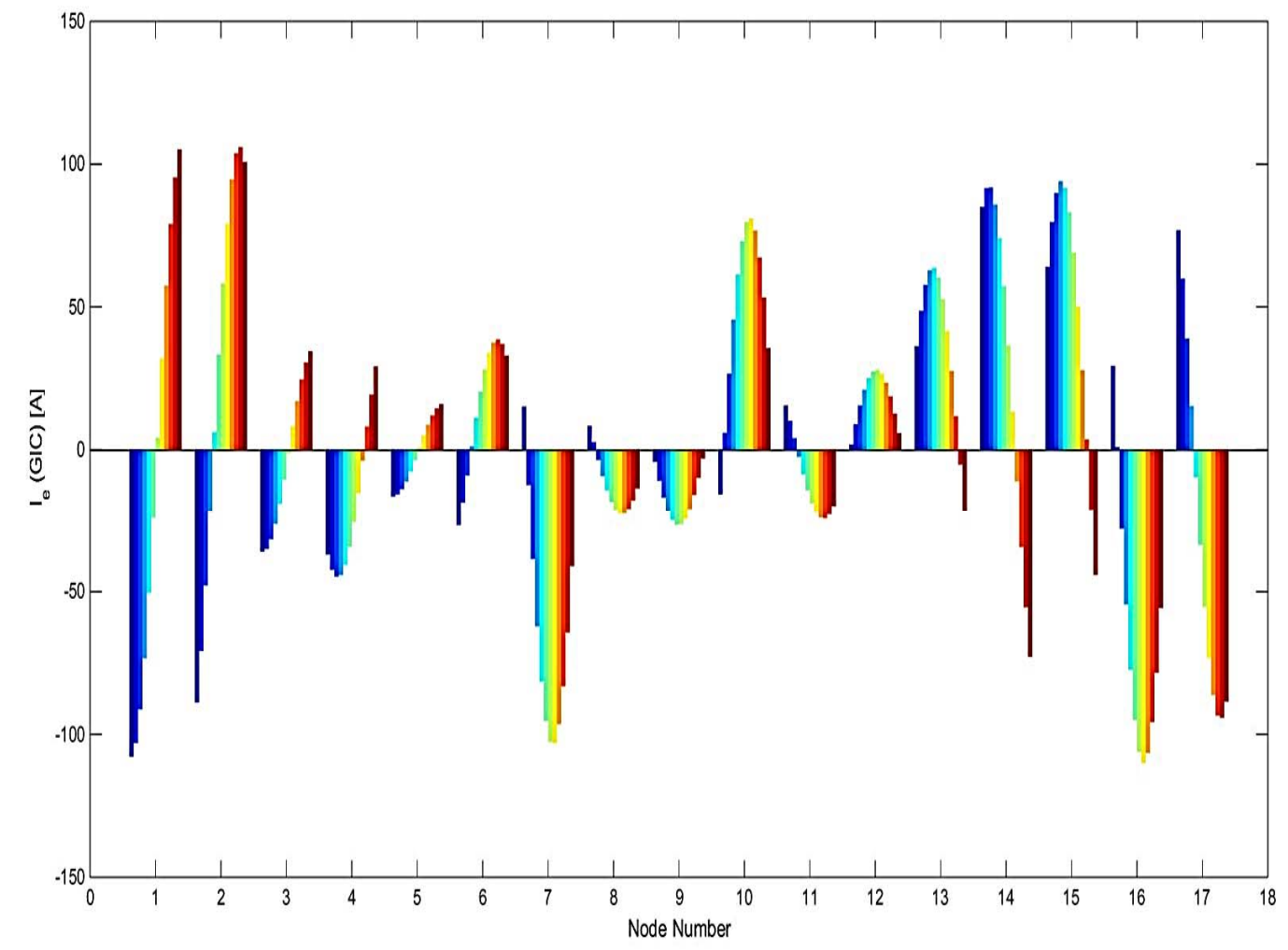

Figure 1. Representation of GICs computed for the stations of the 400-kV Finnish power grid. For each station, the different colors represent a different orientation angle of the geoelectric field $E$.

In this study, the current-flow betweenness of every node $i$ has been computed as the amount of current that flows through $i$ averaged over all the possible paths between source and target nodes ${ }^{21}$. The current betweenness centrality values give a ranking of the relevance of the nodes in current flow transmission in the network (Fig. 2).

20 Freeman L.C., A set of measures of centrality based upon betweenness, Sociometry, 40, 35-41, 1977.

21 Newman M.E.J., A measure of betweenness centrality based on random walks, Social Networks, 27, 39-54, 2005. 


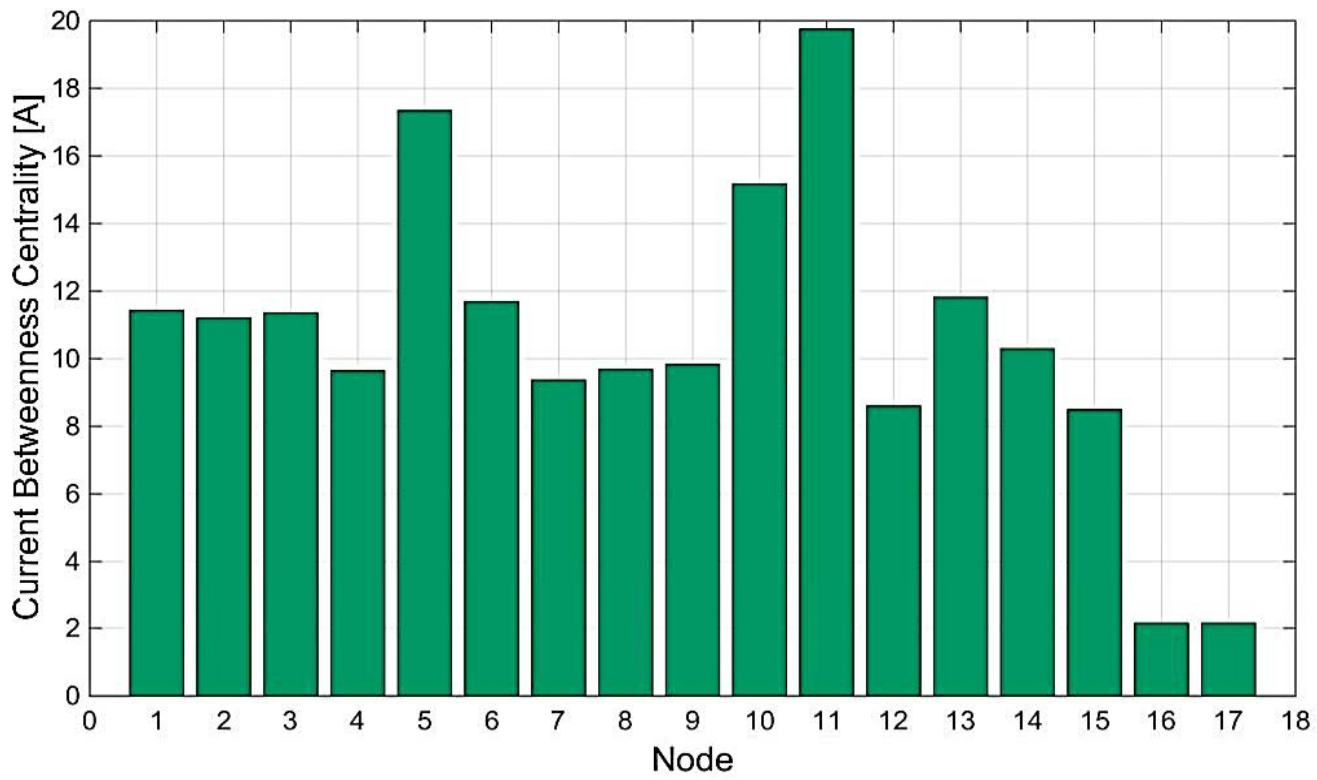

Figure 2. Current betweenness centrality for the stations of the 400-kV Finnish power grid based on the topology, nodes and links, and on the characteristics of the grid, resistances and GICs.

Except for nodes 16 and 17, which are peripheral vertices, the majority of the nodes of the network gives nearly the same contribution to the diffusion of GICs in the network. Nodes with the highest degree (nodes 5, 10 and 13 with degree $\mathrm{k}_{5}=\mathrm{k}_{10}=\mathrm{k}_{13}=3$ and node 11 with $\mathrm{k}_{11}=4$ ) are also nodes with the highest betweenness centrality. These are the elements that mainly affect the spread of GICs throughout the network and should be carefully monitored in planning prevention actions of GIC diffusion during solar storms.

\section{OUTLOOK}

Work is underway to better understand how the structure of the network influences the spreading of space weather effects into the power grid. Consequently, the centrality measures used in network theory will be adapted to embrace also the physical features of the system, for instance, the length of the lines and the location of the nodes. Moreover, the addition of load flow to the induced GIC will also be simulated to understand the resistance of the network to space-weather effects under normal operating conditions. Eventually, this work will be extended to simulate the potential space-weather vulnerability of a simplified European grid. 